MATHEMATICS OF COMPUTATION

Volume 68, Number 225, January 1999, Pages 403-409

S 0025-5718(99)00990-4

\title{
THE NUMBER OF PRIMES $\sum_{i=1}^{n}(-1)^{n-i} i$ ! IS FINITE
}

\author{
MIODRAG ŽIVKOVIĆ
}

\begin{abstract}
For a positive integer $n$ let $A_{n+1}=\sum_{i=1}^{n}(-1)^{n-i} i !, ! n=\sum_{i=0}^{n-1} i$ ! and let $p_{1}=3612703$. The number of primes of the form $A_{n}$ is finite, because if $n \geq p_{1}$, then $A_{n}$ is divisible by $p_{1}$. The heuristic argument is given by which there exists a prime $p$ such that $p \mid ! n$ for all large $n$; a computer check however shows that this prime has to be greater than $2^{23}$. The conjecture that the numbers $! n$ are squarefree is not true because $54503^{2} \mid ! 26541$.
\end{abstract}

Let $N$ and $P$ denote the set of positive integers and the set of prime numbers, respectively. For integers $m, n$ let $(m, n)$ denote their greatest common divisor, and let $m \bmod n$ denote the remainder from division of $m$ by $n$. The fact that $m$ divides (does not divide) $n$ is written as $m \mid n(m \nmid n)$. For $n \geq 2$ let

$$
A_{n+1}=\sum_{i=1}^{n}(-1)^{n-i} i \text { ! }
$$

and let

$$
! n=\sum_{i=1}^{n-1} i !
$$

(the left factorial function was defined by Kurepa [7]). Here we consider the following three questions from [3]. Is it true that

$$
a_{p}:=A_{p} \bmod p \neq 0 \text { for all } p \in P \text { ? }
$$

(This question is raised in connection with [3, Problem B43]: is it true that there are infinitely many prime numbers among $A_{n}, n \in N$.) Is it true that

$$
r_{p}:=! p \bmod p \neq 0 \text { for all } p \in P, \quad p>2 ?
$$

(This is from [3, Problem B44]; an equivalent of the Kurepa hypothesis [7].) And is it true that

$$
\text { for all } n \in N, \quad n>3, \quad ! n \text { is squarefree? }
$$

(This is also in [3, Problem B44]; the second Kurepa hypothesis [7], [9].)

According to [3, Problem B44], R. Bond claims to have proved (2); but he informed this author that he later discovered an error in the proof. Wagstaff verified that (1) and (2) are true for $n<46340$ and $n<50000$, respectively. The calculations were extended by Mijajlović in [9] ((2) for $p \leq 311009)$, Gogić in [2] ((1) and $(2)$ for $p<1000000)$ and Malešević in [8] $((2)$ for $p<3000000)$. Mijajlović proved

Received by the editor July 19, 1996 and, in revised form, January 23, 1997.

1991 Mathematics Subject Classification. Primary 11B83, Secondary 11K31.

Key words and phrases. Prime numbers, left factorial, divisibility.

(c)1999 American Mathematical Society 
in [9] that if $n \in N, p \in P$ and $2<p \leq 1223$, then $! n$ is not divisible by $p^{2}$. A new overview of these questions is given in [4].

For $k \in N$ let $N(k)=\{0,1, \ldots, k-1\}$ and let $R_{k}$ denote the random variable with the uniform probability distribution (PD) over the set $N(k)$. The values $n ! \bmod p$ and $n ! \bmod p^{2}, 1 \leq n<p$, might be considered the independent realizations of $R_{p}$ and $R_{p^{2}}$, respectively (a more precise model could exclude a few boundary values of $n$ ). Consequently, for arbitrary $p \in P$ we can think of $a_{p}$ and $r_{p}$ as the realizations of $R_{p}$.

To check these assumptions, two types of chi-square statistical tests were carried out (for details see, for example, [6, Chapter 3]). The purpose of the first test was to check if given $n=p-1$ integers $z_{i} \in N_{p}, 1 \leq i<p$, might be considered independent realizations of $R_{p}$ (the interesting cases for $z_{i}$ are $i !, !(i+1)$, and $\left.A_{i+1}\right)$. The number $k$ is appropriately chosen and the set $N_{p}$ is divided into $k$ subsets (categories), so that $z_{i}$ belongs to the category $\left[k z_{i} / p\right] \in N_{k}, 1 \leq i<p$ (here $[x]$ denotes the integral part of the real number $x$ ). The frequencies

$$
f_{j}=\left\{i, 1 \leq i<p \mid\left[k z_{i} / p\right]=j\right\}, \quad 0 \leq j<k,
$$

and the expected values $n \pi_{j}, 0 \leq j<k$, can be computed, where

$$
\pi_{j}=\operatorname{Pr}\left(\left[k R_{p} / p\right]=j\right)=\left([p i / k]-[p(i-1) / k]+\delta_{i, 0}-\delta_{i, k-1}\right) / p \simeq 1 / k .
$$

Here $\delta_{i, j}$ is the Kronecker symbol, equal to $1(0)$ if $i=j(i \neq j)$. The frequencies are checked using the $\chi^{2}$ statistics,

$$
\chi^{2}=\sum_{i=0}^{k-1}\left(f_{j}-n \pi_{j}\right)^{2} /\left(n \pi_{j}\right) .
$$

If the value of $\chi^{2}$ is large, then we can say that this experiment contradicts the uniformity of $z_{i}, 1 \leq i<p$. The values of $\chi^{2}$ are calculated with $p$ taking values from a set of random primes (the two first primes following the randomly chosen integer from $\left.\left(2^{l}, 2^{l+1}\right), 10 \leq l \leq 23\right)$, and with $z_{i}$ equal to $i$ !, ! $(i+1)$, and $A_{i+1}$, $1 \leq i<p$, respectively. As expected, the results do not contradict the uniformity assumption. The results for the pairs of consecutive primes do look independent.

The aim of the second type of test is to check the uniformity of the distribution of $a_{p} / p$ and $r_{p} / p$ (when $p \in P$ varies) between the subdivisions

$$
[i / k,(i+1) / k), \quad 0 \leq i<k,
$$

of the unit interval, for some fixed $k$. Let $z_{p}$ denote $a_{p}$ or $r_{p}$. Choose some integers $a<b$ and a prime $p \in P(a, b):=\{p \in P \mid a \leq p<b\}$. For $0 \leq j<k$ compute the frequencies

$$
f_{j}=\left|\left\{p \in P(a, b) \mid\left[k z_{p} / p\right]=j\right\}\right|,
$$

and the category probabilities

$$
\pi_{j}=\operatorname{Pr}\left(\left[k R_{p} / p\right]=j\right) \simeq 1 / k .
$$

Let $n=|P(a, b)|$ be the cardinality of $P(a, b)$. The PD of $R_{p} / p$ over the equal intervals (5) is approximately uniform and independent of $p$. The values of $\chi^{2}(4)$ are computed for $z_{p}=a_{p}, r_{p}$, and $(a, b)=\left(2^{l}, 2^{l+1}\right), 10 \leq l \leq 23(10 \leq l \leq 22$ when $\left.z_{p}=a_{p}\right)$. The results obtained do not contradict the supposed statistical model. 
Using the assumptions about $a_{p}$ and $r_{p}$, we see that (1) and (2) are related to the event

$$
R_{\infty}=\bigcap_{p \in P}\left\{R_{p} \neq 0\right\} .
$$

But according to Mertens's theorem (see [10, Theorem 3.1] for example)

$$
\prod_{p \in P(2, x)}\left(1-\frac{1}{p}\right) \simeq \frac{e^{-\gamma}}{\ln x} \quad \text { as } x \longrightarrow \infty,
$$

where $\gamma$ is Euler's constant, so $e^{-\gamma} \simeq 0.5615$. Therefore, $\operatorname{Pr}\left(R_{\infty}\right)=0$. More precisely, we have the following asymptotic relation

$$
\operatorname{Pr}\left(\bigcap_{p \in P\left(x, x^{\alpha}\right)}\left\{R_{p} \neq 0\right\}\right) \simeq \frac{1}{\alpha}, \quad \text { as } x \longrightarrow \infty .
$$

This heuristic argument suggests that (1) and (2) are not true, and even more, that the number of counterexamples is infinite. The "probability" that there is a counterexample $p \in P\left(x, x^{\alpha}\right)$ to (1) or (2) is approximately $1-1 / \alpha$. With the same probability of $1 / 2$, one counterexample to these claims might be expected in the intervals $\left(2^{3}, 2^{6}\right]=(8,64),\left(2^{6}, 2^{12}\right]=(64,2048]$ and $\left(2^{12}, 2^{24}\right]=(2048,16793216]$. The probability of finding counterexamples in $\left(2^{n}, 2^{n+1}\right]$ is approximately $1 /(n+1)$. The complexity of the search for a counterexample $\leq x$ by the obvious algorithm is $\mathrm{O}\left(x^{2} / \ln x\right)$ [9], which makes it very difficult to check (1) or (2) if, for example, $p>2^{24}$.

The search for values $p \in P$ satisfying $p \mid A_{p}$ was performed using a simple assembler routine for an Intel 80486 microcomputer (at $100 \mathrm{MHz}$ ) calculating $a_{p}$. After approximately 130 hours it was found that for $p=p_{1}=3612703$ we have $p \mid A_{p}$. This fact gives a solution of [3, Problem B43], because for all $n \geq p_{1}$ we have $p_{1} \mid A_{n}$, and so $A_{n}$ is not prime if $n \geq p_{1}$. The numbers $A_{n}$ are prime for $n \in\{4,5,6,7,8,9,11,16,20,42,60,62,106,161\}$. Keller (see [3, Problem B43]) found the last five primes from the list and checked the primality of $A_{n}$ for $n \leq 336$. The necessary condition for primality

$$
3^{M-1} \equiv 1 \quad(\bmod M)
$$

where $M=A_{n}$, is not satisfied if $336<n \leq 563$ (calculations are done using UBASIC [11]), and so the list of known primes $A_{n}$ remains unchanged. By a heuristic argument it could be estimated that if $n<p_{1}$, then $A_{n}$ is prime with the "probability" $2 / n$ (its prime factors are between $n$ and $\sqrt{A_{n}}$ ) and that the total number of primes $A_{n}$ is approximately $2 \ln p_{1} \simeq 30$.

The similar search for values $p \in P$ satisfying $p \mid$ ! $p$, approximately 600 hours long, ended without success. No counterexamples were found to (2) for $p<2^{23}$. The files containing all the residues $a_{p}, p \in P\left(2,2^{22}\right)$ and $r_{p}, p \in P\left(2,2^{23}\right)$, can be obtained from the author on request. An excerpt from the files is given in Table 1 where the instances of $a_{p}$ and $r_{p}$ less than 10 or greater than $p-10$ are listed. Here we see that the congruences $! p \equiv 8(\bmod p)$ and $! p \equiv-7(\bmod p)$ have no solutions $p<2^{23}$. This means that $(! n-8) / 2$ and $! n+7$ are not divisible by any prime less than $2^{23}$; as for $A_{n}$, it is not known whether the number of primes of those two forms is finite (of course, according to the probabilistic model, it is 
TABLE 1. The values of $a_{p}, p<2^{22}$, and $r_{p}, p<2^{23}$, close to 0 or $p$

\begin{tabular}{||r|r||r|r||r|r||r|r||}
\hline \hline$p$ & $a_{p}$ & $p$ & $p-a_{p}$ & $p$ & $r_{p}$ & $p$ & $p-r_{p}$ \\
\hline \hline 2 & 1 & 2 & 1 & 2 & 0 & & \\
3 & 1 & 3 & 2 & 3 & 1 & 3 & 2 \\
5 & 4 & 5 & 1 & 5 & 4 & 5 & 1 \\
7 & 3 & 7 & 4 & 7 & 6 & 7 & 1 \\
11 & 4 & 11 & 7 & 11 & 1 & 13 & 3 \\
17 & 8 & 13 & 1 & 19 & 9 & 17 & 4 \\
31 & 9 & 17 & 9 & 31 & 2 & 23 & 2 \\
41 & 1 & 19 & 5 & 37 & 5 & 67 & 2 \\
43 & 5 & 23 & 5 & 41 & 4 & 71 & 3 \\
47 & 6 & 37 & 1 & 163 & 4 & 113 & 4 \\
67 & 5 & 71 & 7 & 197 & 9 & 139 & 5 \\
79 & 4 & 109 & 5 & 277 & 7 & 227 & 2 \\
157 & 6 & 131 & 3 & 373 & 2 & 349 & 6 \\
191 & 6 & 197 & 2 & 467 & 3 & 2437 & 5 \\
307 & 5 & 229 & 9 & 7717 & 7 & 4337 & 5 \\
641 & 3 & 367 & 4 & 11813 & 6 & 10331 & 2 \\
647 & 5 & 463 & 1 & 33703 & 9 & 77687 & 3 \\
1109 & 2 & 691 & 2 & 2275843 & 3 & 126323 & 8 \\
2741 & 3 & 983 & 3 & 3467171 & 5 & 274453 & 1 \\
3559 & 3 & 1439 & 2 & & & 4709681 & 9 \\
394249 & 1 & 11119 & 3 & & & & \\
2934901 & 1 & 16007 & 4 & & & & \\
3612703 & 0 & 22619 & 3 & & & & \\
& & 32833 & 6 & & & & \\
& & 3515839 & 2 & & & & \\
\hline \hline
\end{tabular}

finite). The check shows that (6) is satisfied by $M=(! n-8) / 2, n \leq 563$ if

$$
n \in\left\{5,6,7,8,11,14,15,16,21,25,48,49,70,108,111,296^{*}\right\},
$$

and that (6) is satisfied by $M=! n+7, n \leq 563$ if

$$
n \in\left\{3,4,5,7,10,12,20,37,52,73,149,304^{*}, 540^{*}\right\} .
$$

Primality of those numbers (excluding the ones with the corresponding $n$ marked by an asterisk) is proved using UBASIC program APRT-CLE [1].

Let $a$ be an arbitrary integer. Consider now divisibilities from [3, Problem B44], i.e. the prime powers $p^{k}(k \geq 1)$ dividing $! n+a$ for all large $n$. For given $p \in P$ and $k \in N$ let

$$
m(p, k)=\min \left\{i \in N\left|p^{k}\right| i !\right\} .
$$

The number $m(p, k)$ is of course a multiple of $p$, and if $k \leq 3$, then $m(p, k)=$ $\left(k-\delta_{p, 2}\right) p$. For all $n \geq m(p, k)$ we have

$$
! n \equiv ! m(p, k) \quad\left(\bmod p^{k}\right) .
$$

Therefore, for all $n \geq m(p, k)$

$$
p^{k} \mid ! n+a \quad \text { iff } \quad p^{k} \mid ! m(p, k)+a .
$$


Especially, if $p>2$ and $k \leq 3$, then for all $n \geq k p$

$$
p^{k} \mid ! n+a \quad \text { iff } \quad p^{k} \mid !(k p)+a .
$$

The case $a=-1$ is considered by Mijajlović and Keller ([3, Problem B44]). Mijajlović noted that $3 \mid ! n-1$ for $n \geq 3,9 \mid ! n-1$ for $n \geq 6$, and $11 \mid ! n-1$ for $n \geq 11$ (by (8) this is the consequence of $3\left|! 3-1,3^{2}\right| ! 6-1$ and $11 \mid ! 11-1$ ). Keller found no new divisibilities of $! n-1$ for $n<10^{6}$. From Table 1 it can be seen that 3 and 11 are the only primes $p<2^{23}$ satisfying $r_{p}=1$, and therefore dividing $! n-1$ for all large $n$. In Table 2 the factorizations of $! n-1, n \leq 42$, (obtained using [5]) are given. The consequence of $11^{2} \nmid !(2 \times 11)-1$ and $3^{3} \nmid !(3 \times 3)-1$ is that $11^{2} \nmid ! n-1$ for $n \geq 22$ and $3^{3} \nmid ! n-1$, for $n \geq 9$. We conclude that $p^{k}=3^{2}$ is the only repeated factor of $! n-1$ for all large $n$ if $p<2^{23}$.

The case $a=0$ is somewhat simpler. Because $r_{p} \neq 0$ for all $p \in P\left(2,2^{23}\right)$, there is not any $p<2^{23}$ such that $p \mid ! n$ for all large $n$. The other cases $-10<a<10$ might be considered similarly using Table 1 .

The other consequence of (7) is that if for the given prime power $p^{k}, k \geq 1$, we are looking for all $n \in N$ such that $p^{k} \mid ! n+a$, then it is enough to check the values of $n \leq m(p, k)$. Let $l$ be the smallest integer satisfying $p^{l} \nmid ! m(p, l)+a$. If $l<k$, then it is enough to check if $p^{k} \mid ! n+a$ for $n<m(p, l) \leq m(p, k)(n<p$ if $l=1$, which is most often the case). Otherwise, if $l \geq k$, then $p^{k} \mid ! m(p, k)+a$ and so $p^{k} \mid ! n+a$ for all $n \geq m(p, k)$. Some repeated factors of $! n-1$ may be seen from Table 2: $3^{4}\left|! 8-1,11^{2}\right| ! 13-1,11^{2} \mid ! 21-1$ and $37^{2} \mid ! 25-1$. By (8) there are no other numbers $! n-1$ divisible by $3^{3}$ or $11^{2}$, because $3^{3} \nmid ! 9-1$ and $11^{2} \nmid ! 22-1$. In Table 3 the triads $(p, n, r)$ are listed satisfying $r=! n \bmod p<10, p \in P\left(2,2^{20}\right)$ and $n \leq 2 p$, except those for which $! n<p$. We see that the only new solution of $p^{2} \mid ! n-1, p<2^{20}, n \in N$, is $41611^{2} \mid ! 26144-1$. From Table 1 we see that $r_{41611} \neq 1$ and consequently $41611 \nmid ! n-1$ for $n \geq 41611$.

Table 3 contains a counterexample to (3): the relation $54503^{2} \mid ! 26541$ shows that left factorials are not always squarefree. The existence of a counterexample also has a "probabilistic" explanation. Considering the values $! n \bmod p^{2}, 1 \leq n \leq p$, as the independent realizations of $R_{p^{2}}$, the check of $! n \bmod p^{2} \neq 0,1 \leq n \leq p$, for fixed $p \in P$ corresponds to the event $T_{p}$ that $p$ independent outcomes of $R_{p^{2}}$ are all different from 0 . Using the inequality

$$
1-\frac{1}{n}<\left(1-\frac{1}{n^{2}}\right)^{n}<\left(1-\frac{1}{n}\right) /\left(1-\frac{1}{n^{2}}\right),
$$

which can be easily proved, we conclude that

$$
\operatorname{Pr}\left(T_{p}\right)=\left(1-1 / p^{2}\right)^{p} \simeq 1-1 / p
$$

for large $p$. It follows that (3) and (1) have the same asymptotic "counterexample densities".

The seemingly unexpected repetitions in Table 3 can be explained as follows. The remainders $! n \bmod p^{2}, p \leq n \leq 2 n$, have the same remainder $\bmod p$. Therefore, if ! $p \bmod p<10$ (hence this $p$ appears in Table 1 ), then with the high "probability" of $(1-1 / p)^{p-1} \simeq e^{-1}$ there will be exactly one such entry $(p, n, r)$ in Table 3 ; furthermore, with the "probability" of $\left(\begin{array}{l}p \\ 2\end{array}\right) 1 / p^{2}(1-1 / p)^{p-2} \simeq 0.5 e^{-1}$ there will be two entries $(p, n, r)$ and $\left(p, n^{\prime}, r\right)$ with the same small remainder. Even the probability of three entries differing only in the second position is not too small, 
TABLE 2 . The factorizations of $! n-1, n \leq 42$

\begin{tabular}{||r|l||}
\hline \hline$n$ & The factorization of !n-1 \\
\hline \hline 3 & 3 \\
4 & $3^{2}$ \\
5 & $3 \times 11$ \\
6 & $3^{2} \times 17$ \\
7 & $3^{2} \times 97$ \\
8 & $3^{4} \times 73$ \\
9 & $3^{2} \times 11 \times 467$ \\
10 & $3^{2} \times 131 \times 347$ \\
11 & $3^{2} \times 11 \times 40787$ \\
12 & $3^{2} \times 11 \times 443987$ \\
13 & $3^{2} \times 11^{2} \times 23 \times 20879$ \\
14 & $3^{2} \times 11 \times 821 \times 83047$ \\
15 & $3^{2} \times 11 \times 2789 \times 340183$ \\
16 & $3^{2} \times 11 \times 107 \times 509 \times 259949$ \\
17 & $3^{2} \times 11 \times 225498914387$ \\
18 & $3^{2} \times 11 \times 163 \times 20143 \times 1162943$ \\
19 & $3^{2} \times 11 \times 19727 \times 3471827581$ \\
20 & $3^{2} \times 11 \times 29 \times 43 \times 1621 \times 641751001$ \\
21 & $3^{2} \times 11^{2} \times 53 \times 67 \times 662348503367$ \\
22 & $3^{2} \times 11 \times 877 \times 3203 \times 41051 \times 4699727$ \\
23 & $3^{2} \times 11 \times 11895484822660898387$ \\
24 & $3^{2} \times 11 \times 139 \times 2129333 \times 922459185301$ \\
25 & $3^{2} \times 11 \times 37^{2} \times 29131483 \times 163992440081$ \\
26 & $3^{2} \times 11 \times 454823 \times 519472957 \times 690821017$ \\
27 & $3^{2} \times 11 \times 107 \times 173 \times 7823 \times 12227 \times 1281439 \times 1867343$ \\
28 & $3^{2} \times 11 \times 431363 \times 2882477797 \times 91865833117$ \\
29 & $3^{2} \times 11 \times 191 \times 47793258077 \times 349882390108241$ \\
30 & $3^{2} \times 11 \times 37 \times 283 \times 5087 \times 1736655143086866180331$ \\
31 & $3^{2} \times 11 \times 2771826449193354891007108898387$ \\
32 & $3^{2} \times 11 \times 1231547 \times 306730217 \times 227214279676815713$ \\
33 & $3^{2} \times 11 \times 41 \times 163 \times 224677 \times 278437 \times 6562698554476756561$ \\
34 & $3^{2} \times 11 \times 109 \times 839 \times 2819 \times 40597679 \times 8642572321688037037$ \\
35 & $3^{2} \times 11 \times 3072603482270933019578343003268898387$ \\
36 & $3^{2} \times 11 \times 7523968684626643 \times 14280739323850758510209$ \\
37 & $3^{2} \times 11 \times 542410073 \times 7125524357434108671946525659019$ \\
38 & $3^{2} \times 11 \times 379 \times 2677 \times 5685998930867 \times 24769422762368668966567$ \\
39 & $3^{2} \times 11 \times 127 \times 338944799 \times 126050058872020979628982810240819$ \\
40 & $3^{2} \times 11 \times 956042657 \times 221187999196843747210838711867563891$ \\
41 & $3^{2} \times 11 \times 8453033680104197032254976173172281742468898387$ \\
42 & $3^{2} \times 11 \times 1652359939 \times 276306566079013 \times 758627421394906687355741$ \\
\hline \hline & \\
\hline
\end{tabular}

approximately $e^{-1} / 6$. Otherwise, if $! p \bmod p>10$, then

$$
\left(! n \bmod p^{2}\right) \bmod p=! p \bmod p>10 \text { for } p \leq n \leq 2 p,
$$

and therefore there cannot be an entry $(p, \cdot, \cdot)$ in Table 3 . 
TABLE 3 . The small values of $! n \bmod p^{2}<10$, for $p \in P, p<2^{20}$, $1 \leq n \leq 2 p$

\begin{tabular}{|r|r|c||r|r|c||}
\hline \hline$p$ & $n$ & $! n \bmod p^{2}$ & $p$ & $n$ & $! n \bmod p^{2}$ \\
\hline \hline 2 & 3 & 0 & 83 & 60 & 5 \\
2 & 4 & 2 & 163 & 183 & 4 \\
3 & 4 & 1 & 163 & 273 & 4 \\
3 & 5 & 7 & 173 & 152 & 3 \\
3 & 6 & 1 & 197 & 355 & 9 \\
5 & 5 & 9 & 373 & 185 & 6 \\
5 & 6 & 4 & 373 & 514 & 2 \\
5 & 9 & 9 & 467 & 730 & 3 \\
7 & 6 & 7 & 467 & 902 & 3 \\
11 & 13 & 1 & 3119 & 306 & 6 \\
11 & 21 & 1 & 4357 & 837 & 7 \\
17 & 7 & 7 & 7717 & 9402 & 7 \\
17 & 11 & 6 & 7717 & 15415 & 7 \\
19 & 17 & 9 & 8297 & 4727 & 7 \\
19 & 20 & 9 & 33703 & 39795 & 9 \\
37 & 25 & 1 & 33703 & 43801 & 9 \\
37 & 63 & 5 & 33703 & 52337 & 9 \\
41 & 55 & 4 & 41611 & 26144 & 1 \\
43 & 9 & 9 & 54503 & 26541 & 0 \\
47 & 19 & 8 & 302837 & 283148 & 8 \\
59 & 41 & 9 & 351731 & 135646 & 8 \\
67 & 29 & 8 & & & \\
\hline \hline
\end{tabular}

\section{REFERENCES}

1. K. Akiyama, Y. Kida, F. O'Hara, APRT-CLE, Cohen-Lenstra version of Adleman-PomeranceRumely Test, UBASIC program, 1988-1992.

2. G. Gogić, Parallel algorithms in arithmetic, Master thesis, Belgrade University, 1991.

3. R. Guy, Unsolved problems in number theory, Second Edition, Springer-Verlag, 1994. MR 96e: 11002

4. A. Ivić, Ž. Mijajlović, On Kurepa's problems in number theory, Publ. Inst. Math. (Beograd) (N. S.), 57(71), 1995, 19-28 MR 97a:11007

5. Y. Kida, ECMX, Prime Factorization by ECM, UBASIC program, 1987-1990.

6. D. E. Knuth, The Art of Computer Programming, Vol. 2 Addison-Wesley, Reading 1969. MR 44:3531

7. Dj. Kurepa, On the left factorial function, Math. Balkanica, 1, 1971, 147-153. MR 44:3945

8. B. Malešević, Personal communication.

9. Ž. Mijajlović, On some formulas involving $! n$ and the verification of the $! n$ hypothesis by use of computers, Publ. Inst. Math. (Beograd), 47(61), 1990, 24-32. MR 92d:11134

10. H. Riesel, Prime numbers and computer methods for factorization, Birkhauser, Boston, 1985. MR 88k: 11002

11. UBASIC, version 8.74, 1994.

MatematičKi Fakultet, Beograd

E-mail address: ezivkovm@@matf.bg.ac.yu 